\title{
Determination of the Intracellular Concentrations of Metabolites in Escherichia coli Collected during the Exponential and Stationary Growth Phases using Liquid Chromatography-Mass Spectrometry
}

\author{
Changhun Park, Changhun Park, ${ }^{\dagger, \dagger}$ Youn-jin Lee, ${ }^{\dagger}$ Sang Yup Lee, ${ }^{\S}$ Han Bin Oh, ${ }^{\dagger, *}$ and Jinwon Lee ${ }^{*}$ \\ Department of Chemical and Biomolecular Engineering, Sogang University, Seoul 121-742, Korea \\ *E-mail: jinwonlee@sogang.ac.kr \\ ${ }^{\dagger}$ Department of Chemistry, Sogang University, Seoul 121-742, Korea. *E-mail: hanbinoh@sogang.ac.kr \\ ${ }^{\ddagger}$ Clinical Trials Center, Yonsei University Health System, Seoul 120-725, Korea \\ ${ }^{\S}$ Department of Chemical and Biomolecular Engineering, Korea Advanced Institute of Science and Technology, \\ Daejeon 305-701, Korea \\ Received September 30, 2010, Accepted December 3, 2010
}

\begin{abstract}
In the present study, we demonstrate that SRM LC-MS/MS method developed by Luo et al. (ref. 10) can be successfully applied to the quantitative analysis of intracellular metabolites in E. coli that are collected at the exponential and stationary growth phases. A focus is given on measuring the changes in the concentrations of intracellular metabolites in batch cultures, which were induced during both the dynamically changing exponential and stationary growth phases. The following intracellular metabolites are quantified in the exponential and stationary phases of E. coli growth, using the SRM mode of a triple quadrupole mass spectrometer: glucose-1-phosphate, fructose-1,6-bisphosphate, phosphoenolpyruvate, pyruvate, acetyl-coenzyme A, 6-phosphogluconate, ribulose-5-phosphate, xylulose-5-phosphate, erythrose-4-phosphate. The determined intracellular metabolite concentration profiles are shown to be in a good agreement with the growth profiles of $E$. coli, which clearly indicates that SRM LC-MS/MS can be successfully used for following the metabolite changes induced at different growth stages.
\end{abstract}

Key Words: Metabolomics, SRM, E. Coli, LC-MS, Metabolites

\section{Introduction}

Metabolomics, a comprehensive, quantitative analysis of metabolites in a biological system, has greatly enhanced the understanding of biological phenomena and systems. ${ }^{1-4}$ Along with other -omics technologies such as genomics, transcriptomics, and proteomics, metabolomic investigations have increased integrative knowledge of biochemical and biological mechanism in complex systems. ${ }^{5}$ Since metabolomics represents the endpoint of the -omics cascade, it is the closest to phenotype, and its application is now rapidly expanding., 5

In recent years, studies of cellular systems have become increasingly active and productive, due to the recent advance in the optimization of both cellular growth and the production process. Cellular growth is primarily enhanced by metabolic engineering, and improvements in productivity are primarily accomplished through the advancements in bioprocess engineering. ${ }^{7}$ To enhance cellular growth, understanding of the central carbon metabolism (e.g., glycolysis, the pentose phosphate pathway, and the TCA cycle) is crucial because these pathways are closely involved in biosynthetic reactions, energy metabolism, and anaplerotic reactions. ${ }^{7}$ The understanding of the central carbon metabolism, for example, of E. coli, and its related in vivo kinetics can provide us with a framework with which a stream of modifications of intermediary metabolisms can be made to increase the biosynthetic production of primary and secondary metabolites. $^{7}$

A detailed understanding of the central carbon metabolism can be achieved through the quantification of the metabolites involved in their pathways. Measurements of metabolite con- centrations provide quantitative information concerning the kinetics of metabolism; these measurements can be used for mathematical modeling. Mass spectrometry has emerged as a key technology for the quantitative analysis of metabolites. ${ }^{7,8}$ Mass spectrometry is very useful for the analysis of extracellular and intracellular metabolites due to its extraordinary sensitivity, low sample consumption, and high selectivity. Gas chromatography-mass spectrometry (GC-MS) has been described as the gold standard for metabolomic research. ${ }^{8}$ This tool is becoming increasingly popular due to its ability of chromatographic separation, analyte-specific detection, quantification, and identification of unknown metabolites. ${ }^{5,8,9}$ However, this method requires additional chemical derivatization procedures to reduce the polarity and to increase the volatility and thermal stability of the metabolites under examination.

In contrast, electrospray ionization mass spectrometry (ESIMS) has many advantages over GC-MS because ESI-MS does not require the chemical derivatization of samples. Furthermore, when coupled with chromatography, this method can provide additional dimensionality. Chromatographic separation even makes it possible to distinguish isomers, which produce very distinctive MS/MS patterns. ${ }^{10}$

The most difficult types of metabolites to analyze are high polarity metabolites, such as nucleotides and sugar phosphates, due to their highly ionic nature. ${ }^{11}$ To analyze these types of metabolites, anion exchange chromatography and ion pairing chromatography-mass spectrometry are reported to be useful. ${ }^{10-14}$ Most recently, Luo et al. developed a highly selective and sensitive ion pairing-liquid chromatography-mass spectrometry method for the simultaneous identification and quantification 


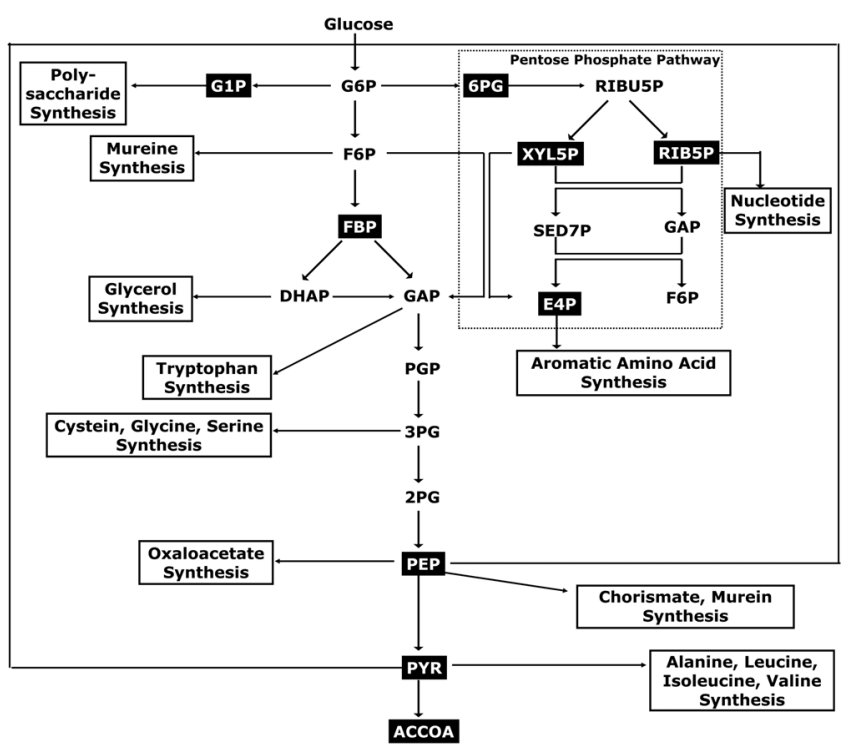

Scheme 1. Glycolysis metabolic pathway and pentose phosphate pathway in E. coli; metabolites examined in the present study are denoted in filled boxes

of 29 negatively charged compounds including sugar phosphates, nucleotides, and carboxylic acids by using a $\mathrm{C} 18$ reversed-phase column. ${ }^{10}$ They employed tributylammonium acetate as the volatile ion pair modifier, resulting in low limits-of-detection (LOD).

In the present study, we demonstrate that the above-mentioned method developed by Luo et al. can be successfully applied to the quantitative analysis of intracellular metabolites in E. coli, which are collected at the exponential and stationary phases of growth. ${ }^{10}$ Examination of the metabolite concentration profile changes at different growth phases is an interesting subject in metabolomics. More specifically, measurements of intracellular metabolites at various growth stages are crucial for the development of mathematical models. This kinetic modeling is currently underway and will be presented in a forthcoming paper. In this study, measurements were made for the following selected metabolites that are involved in the central carbon metabolism; G1P (Glucose-1-phosphate), FBP (Fructose-1,6-bisphosphate), PEP (Phosphoenolpyruvate), PYR (Pyruvate), AcCoA (Acetyl-coenzyme A), 6PG (6-Phosphogluconate), Ri5P (Ribulose-5-phosphate), X5P (Xylulose-5-phosphate), and E4P(Erythrose-4phosphate). These metabolites are key intermediates in glycolysis metabolic pathway and pentose phosphate pathway (refer to Scheme 1). ${ }^{15}$

\section{Materials and Methods}

Chemicals. E. coli K-12 strain W3110 (KCTC 2223) was obtained from the BRC (Biological resource center in Korea). Extracellular and intracellular metabolite standards were purchased from Sigma and Fluka. High purity solvents and reagents were used to avoid the appearance of interfering MS peaks and high background. Tributylamine was obtained from Aldrich and acetic acid (GC standard grade) was purchased from Fluka. The solvents used for chromatography were of HPLC grade.
Culture Condition. Precultivation was performed overnight $\left(12 \mathrm{~h}, 200 \mathrm{rpm}, 37^{\circ} \mathrm{C}\right)$ in a shake flask $(500 \mathrm{~mL})$ containing $200 \mathrm{~mL}$ of precultivation medium (LB medium). E. coli K-12 strain W3110 was batch cultivated in a stirred bioreactor (150 rpm) with a working volume of $2.0 \mathrm{~L}$ at $37^{\circ} \mathrm{C}$. Synthetic mineral medium was used for the batch culture. The concentrations of components in the synthetic mineral medium included: $4.0 \mathrm{~g} / \mathrm{L} \mathrm{Na}_{2} \mathrm{SO}_{4} ; 5.36 \mathrm{~g} / \mathrm{L}\left(\mathrm{NH}_{4}\right)_{2} \mathrm{SO}_{4} ; 1.0 \mathrm{~g} / \mathrm{L} \mathrm{NH}_{4} \mathrm{Cl} ; 7.3 \mathrm{~g} / \mathrm{L}$ $\mathrm{K}_{2} \mathrm{HPO}_{4} ; 1.8 \mathrm{~g} / \mathrm{L} \mathrm{NaH} \mathrm{PO}_{4} \mathrm{H}_{2} \mathrm{O} ; 12.0 \mathrm{~g} / \mathrm{L}\left(\mathrm{NH}_{4}\right)_{2}$-H-citrate; $4.0 \mathrm{~mL} / \mathrm{L} \mathrm{MgSO}_{4}(1 \mathrm{M}) ; 6.0 \mathrm{~mL} / \mathrm{L}$ trace element solution; $0.02 \mathrm{~g} / \mathrm{L}$ thiamine; and $20.0 \mathrm{~g} / \mathrm{L}$ glucose. The air flow was kept constant at $2 \mathrm{~L} / \mathrm{min}$ and the $\mathrm{pH}$ was controlled at 7.0 by the addition of $2 \mathrm{M} \mathrm{NaOH}$. To prevent foam formation, an anti-foam agent was added periodically.

Sampling, Quenching, and Extraction. Samples were taken every $2 \mathrm{~h}$ after batch culture initiation. The culture sample vials $(5 \mathrm{~mL})$ were plunged rapidly into an equal volume of $60 \%$ aqueous methanol $\left(-40^{\circ} \mathrm{C}\right)$. The quenched biomass was centrifuged for $8.5 \mathrm{~min}$ (i.e., the minimum time period required to form a pellet) at $3,000 \times g$ and $-9{ }^{\circ} \mathrm{C}$. The supernatant was removed rapidly, and an aliquot $(1 \mathrm{~mL})$ was retained to assess the leakage of internal metabolites. The pellets and supernatants were frozen in liquid nitrogen and stored at $-80{ }^{\circ} \mathrm{C}$ for further analysis. ${ }^{16}$ The first step of the methanol extraction method froze the pellets. The biomass pellets were suspended in $500 \mu \mathrm{L}$ of $100 \%$ methanol $\left(-40^{\circ} \mathrm{C}\right)$ and frozen in liquid nitrogen. The second step was thawing. The biomass pellets were allowed to thaw on dry ice. The freeze-thaw rotation was performed three times to cause the metabolites to leak from the cells. The suspensions were then centrifuged at $16,000 \times g$ at $-9{ }^{\circ} \mathrm{C}$ for $5 \mathrm{~min}$. The supernatant was retained and stored on dry ice, and a $500 \mu \mathrm{L}$ aliquot of $100 \%$ methanol $\left(-48{ }^{\circ} \mathrm{C}\right)$ was then added to the pellet. The above procedure was repeated, and the two aliquots were combined. The sample was stored at $-80{ }^{\circ} \mathrm{C} .{ }^{17}$

Measurement of Extracellular Metabolites Concentration. The concentrations of glucose, lactate, formate and acetate were analyzed using a high-performance liquid chromatography (HPLC, UV 730D detector, RI 750F monitor; Younglin, Korea) equipped with an ion-exchange column (Aminex HPX-87H, $300 \mathrm{~mm} \times 7.8 \mathrm{~mm}$; Bio-Rad) using $0.005 \mathrm{~N} \mathrm{H}_{2} \mathrm{SO}_{4}$ as the mobile phase at $55{ }^{\circ} \mathrm{C}$ and a flow rate of $0.6 \mathrm{~mL} / \mathrm{min}$.

LC-MS/MS Measurement. All LC-MS/MS experiments were carried out using an Applied Biosystems/MDS Sciex API 3200 triple quadrupole mass spectrometer (AB/MDS Sciex, Concord, Canada) equipped with a turbo-ion spray source. The mass spectrometer was coupled with an Agilent 1200 series HPLC system (Agilent Technologies, Santa Clara CA, USA). Data were acquired and processed using Analyst software (version 1.4.2, AB/ MDS Sciex, Concord, Canada). The mass spectrometer was operated in the negative ion and selected reaction monitoring (SRM) modes. The declustering potential (DP), collision energy (CE) and collision cell exit potential (CXP) were carefully optimized. The entrance potential (EP) was fixed at $-10 \mathrm{~V}$ throughout all experiments. The MS parameters were as follow: the ionspray voltage was $-4,500 \mathrm{~V}$, the nebulizer gas (GS1), auxiliary gas (GS2), curtain gas (CUR) and collision gas (CAD) values were 20,20, 10, and 5 (arbitrary units), respectively. The auxiliary gas temperature was maintained at $500{ }^{\circ} \mathrm{C}$. The chro- 
Table 1. Gradient profile used in the LC-MS/MS method

\begin{tabular}{cccc}
\hline Step & Total time (min) & Eluent A (vol \%) & Eluent B (vol \%) \\
\hline 1 & 15.00 & 100.0 & 0.0 \\
2 & 25.00 & 80.0 & 20.0 \\
3 & 55.00 & 80.0 & 20.0 \\
4 & 60.00 & 65.0 & 35.0 \\
5 & 65.00 & 65.0 & 35.0 \\
6 & 70.00 & 40.0 & 60.0 \\
7 & 75.00 & 40.0 & 60.0 \\
8 & 75.10 & 10.0 & 90.0 \\
9 & 80.00 & 10.0 & 90.0 \\
\hline
\end{tabular}

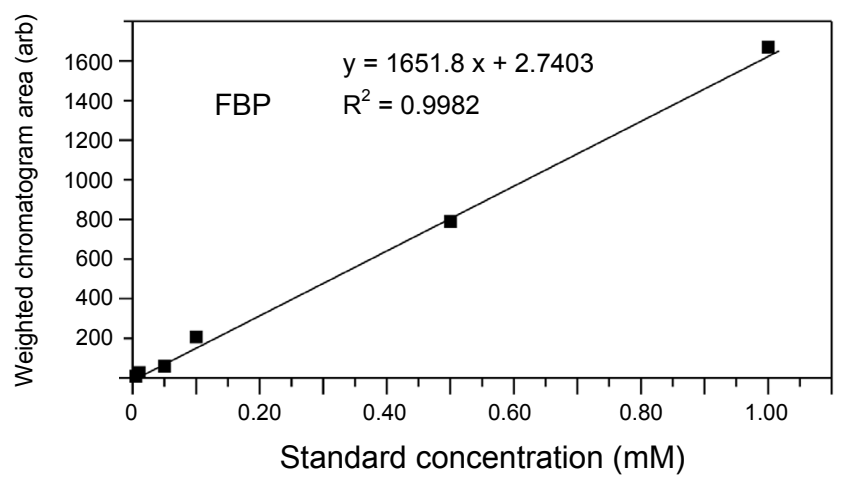

Figure 1. The weighted calibration curve obtained for FBP.

matographic separation was achieved with a Synergi Hydro$\mathrm{RP}(\mathrm{C} 18) 150 \mathrm{~mm} \times 2.1 \mathrm{~mm}$ I.D., $4 \mu \mathrm{m} 80 \AA$ A particles column (Phenomenex, Torrance, CA, USA) at room temperature. Eluent A was a $10 \mathrm{mM}$ aqueous tributylamine solution with its $\mathrm{pH}$ adjusted to $\mathrm{pH} 4.95$ using $15 \mathrm{mM}$ acetic acid, and eluent $\mathrm{B}$ was methanol. A binary gradient at the flow rate of $0.2 \mathrm{~mL} / \mathrm{min}$ is shown in Table 1.

Quantification. Quantitative analysis was made using 'weighted' regression analysis method. ${ }^{18}$ Primary stock aqueous solutions were prepared at a concentration of $5 \mathrm{mM}$ for each metabolite. From this solution one stock solution of $100 \mu \mathrm{M}$ standard mixture was prepared in water. Aliquots of this standard mixture were used for calibration and for the quality control (QC) standards. The calibration curve was obtained by analyzing standard solutions at six concentrations, ranging from $0.005 \mathrm{mM}$ to $1 \mathrm{mM}(0.005,0.01,0.05,0.1,0.5$, and $1 \mathrm{mM})$. The calibration curves were constructed by plotting the SRM chromatogram area of the compound (weighted to $1 / x$, where $x$ is the concentration in $\mathrm{mM}$ ) against the concentration of the compound (x). For example, Figure 1 shows the weighted calibration curve obtained for FBP. Weighted linear regression was used to fit the calibration curve and the linearity for each compound was evaluated using the calibration curve.

\section{Results and Discussion}

Growth Profile of the Batch Culture. The growth profile of the $E$. coli batch culture was obtained at 2 hour intervals for up to 18 hours by measuring the dry weights of the biomass and the concentrations of the extracellular metabolites (see Figure 2). The dry weights of the biomass (in $\mathrm{g} / \mathrm{L}$ ) were determined by

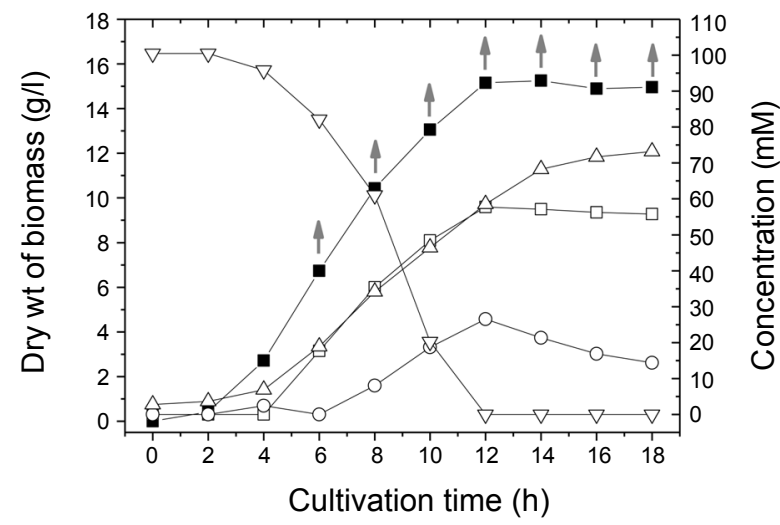

Figure 2. Growth profiles of bioreactor batch cultures: (घ) Dry weight of the biomass. The upward arrow indicates the time at which samples were taken for intracellular metabolite analyses. Extracellular metabolite concentration profile: $(\nabla)$ Glucose, $(\triangle)$ Acetate, $(\square)$ Formate, and $(\circ)$ Lactate.

measuring the optical transmittance of the collected culture media at $600 \mathrm{~nm}$; these values are denoted as solid squares in Figure 2. The concentrations of the four extracellular metabolites, glucose $(\nabla)$, acetate $(\triangle)$, formate $(\square)$, and lactate $(\circ)$, which will be determined using SRM quantitative analysis, are also shown in Figure 2. These dry weights indicate that the $E$. coli grew exponentially for up to 12 hours before reaching the stationary phase. Similar production profiles were seen for "growth-associated" products, ${ }^{19}$ i.e., acetate, formate, and lactate. After $\sim 12 \mathrm{~h}$, the "growth-associated" metabolites showed a short period of arithmetic growth before the cells entered the stationary phase. Interestingly, the end of the exponential phase coincided with the almost complete consumption of glucose in the extracellular medium by E. coli.

Quantitative Analysis of Intracellular Metabolites. For quantitative analysis of intracellular metabolites, the MS and MS/ MS experimental parameters were optimized for the standard metabolite samples using directly infused electrospray ionization mass spectrometry (ESI-MS); the standard samples examined in this study included G1P (Glucose-1-phosphate), FBP (Fructose-1,6-bisphosphate), PEP (Phosphoenolpyruvate), PYR (Pyruvate), AcCoA (Acetyl-coenzyme A), 6PG (6-Phosphogluconate), Ri5P (Ribulose-5-phosphate), X5P (Xylulose-5phosphate), and E4P (Erythrose-4-phosphate). The detection of the metabolites was made using the negative-ion mode because all of the metabolites of interest have low $\mathrm{p} K_{\mathrm{a}}$ values. All of the metabolites examined in this study have a phosphate group in common, except for PYR, and thus their collision induced dissociation (CID) MS/MS spectra shared similar fragmentation characteristics.

Mass Spectrometric Analysis. As shown in Figure 3, $\left[\mathrm{PO}_{3}\right]$ or $\left[\mathrm{H}_{2} \mathrm{PO}_{4}\right]^{-}$was a dominant CID MS/MS metabolic product, except when PYR were analyzed. For PYR, decarboxylated precursor ions were found at $m / z 43$. It is important to note that Ri5P and X5P are isobaric compounds (detected at $\mathrm{m} / \mathrm{z} 229$ ) differing only in the stereochemistry at $\mathrm{C} 3$. They are indistinguishable by MS/MS due to their structural similarities. These observations are summarized in Table 2, where the $\mathrm{m} / \mathrm{z}$ values of metabolites of interest, $[\mathrm{M}-\mathrm{H}]^{-}$, and their main product ions 
(a)

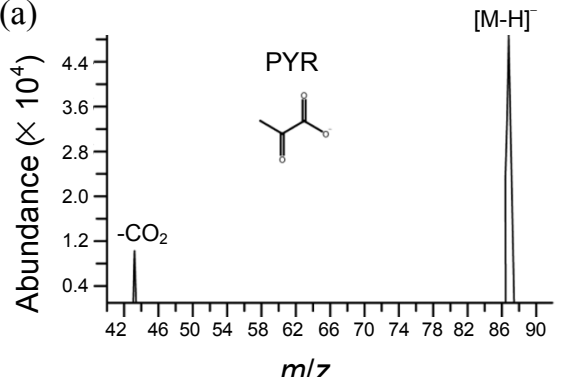

(d)

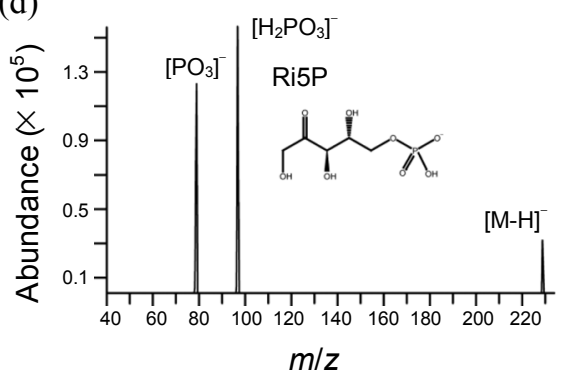

(g)

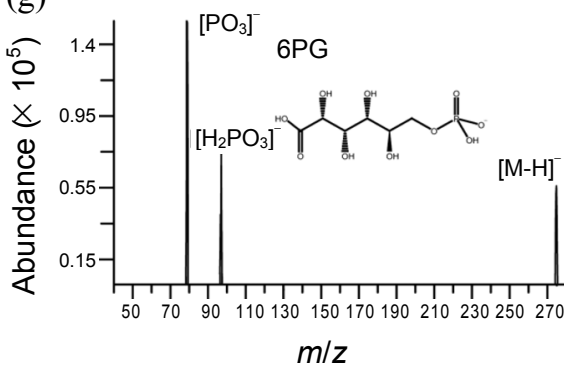

(b)

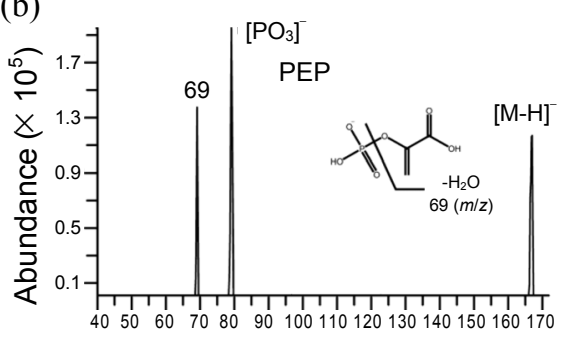

$\mathrm{m} / \mathrm{z}$

(e)

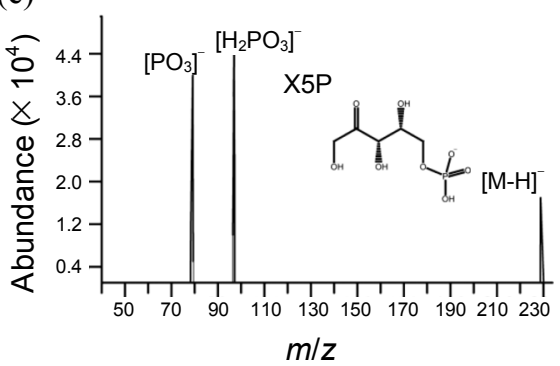

(h)

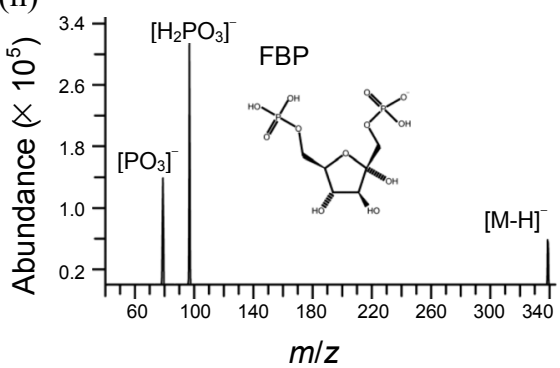

(c)

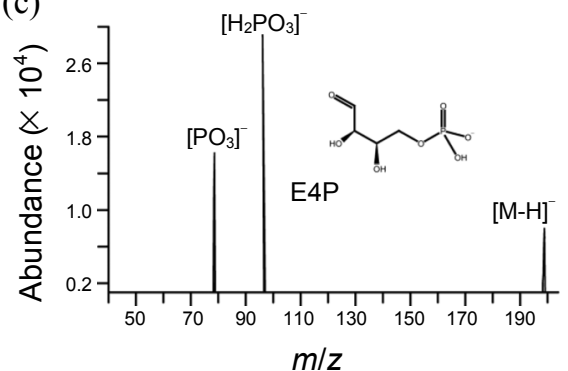

(f)

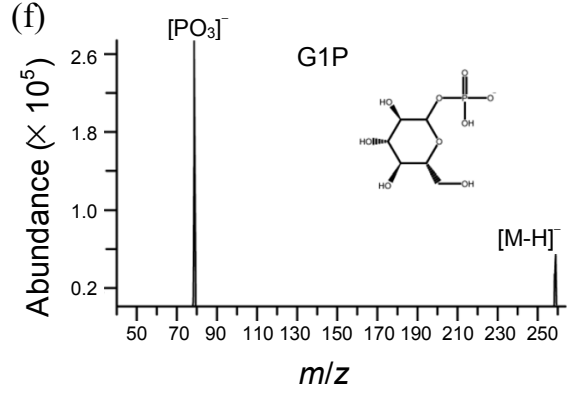

(i)

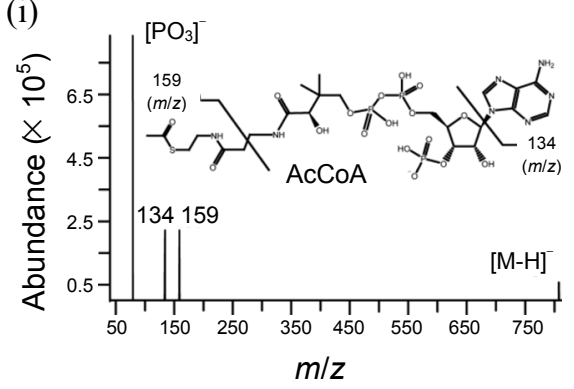

Figure 3. Collision induced dissociation (CID) product mass spectrometry spectrum: (a) PYR standard; (b) PEP standard; (c) E4P standard; (d) Ri5P standard; (e) X5P standard; (f) G1P standard; (g) 6PG standard; (h) FBP standard; and (i) AcCoA standard.

are indicated. The experimental parameters for mass spectrometry are also indicated in the table. The main products shown in Table 2 were used as the SRM transitions in the mass spectrometric quantifications.

The metabolites were quantified using a triple-quadrupole mass spectrometer in the SRM mode. The liquid chromatography parameters used in this study, such as the gradient profile and ion-pairing reagents, were the same as those used by Luo et al..$^{10}$ These authors found that tributylamine (TBA) was an optimal ion-pairing agent in terms of separation resolution and retention time, compared to other trialkylamines. Furthermore, they found that $\mathrm{pH}$ was also crucial for optimal chromatographic peak resolution; $\mathrm{pH}$ value of 4.95 was shown to be best. Therefore, a $10 \mathrm{mM}$ aqueous TBA solution adjusted to $\mathrm{pH} 4.95$ was used in this study (see Materials and Methods).

Chromatographic Analysis. Figure 4 shows selected ion chromatograms (SICs) for the nine metabolites that were obtained from the standard mixture. It was crucial to obtain retention times for the sample metabolites. Knowledge of both the specific SRM transition and the retention time is required for quantitative metabolite analysis because it can minimize the chance of producing a false positive reading. The elution order shown in Figure 4 is consistent with that of Luo et al., although there were some differences in specific retention times. ${ }^{10}$ The reten- tion time for each metabolite was used for further analysis of the intracellular metabolites.

The limits of detection (LOD) were also determined for the metabolites by taking three times the standard deviation of the background signals. The obtained LODs ranged from 0.01 to $0.24 \mu \mathrm{M}$, except for $2.27 \mu \mathrm{M}$ for PYR (see Table 3). For PYR, the relatively poor product ion signal at $m / z 43$ limited the LODs; these results are in agreement with those of Luo et al. ${ }^{10}$ The LODs obtained in this study were approximately one order of magnitude higher than those of Luo et al., ${ }^{10}$ but they were approximately the same order of magnitude as those obtained in other studies. ${ }^{12,14,20}$ The higher LODs were presumably due to the use of a less sensitive mass spectrometer (Applied Biosystems/MDS Sciex API 3200 triple quadrupole mass spectrometer versus $4000 \mathrm{Q}$ TRAP triple quadrupole mass spectrometer). Furthermore, our sample injection volume was rather small, i.e., $1 \mu \mathrm{L}$.

Intracellular Metabolite Concentration Profiles at Two Different Growth Phases. The concentrations of intracellular metabolites at various $E$. coli growth phases were determined using the weighted regression analysis method described in the experimental section. ${ }^{18}$ The $E$. coli growth phase, which is reflected in the dry weight of the biomass, is indicated by solid squares in Figure 2. For intracellular metabolite analyses, $5 \mathrm{~mL}$ sam- 
(a)

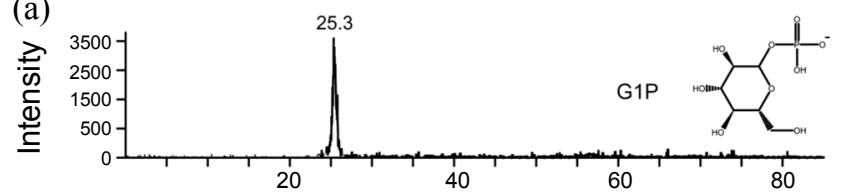

(b)

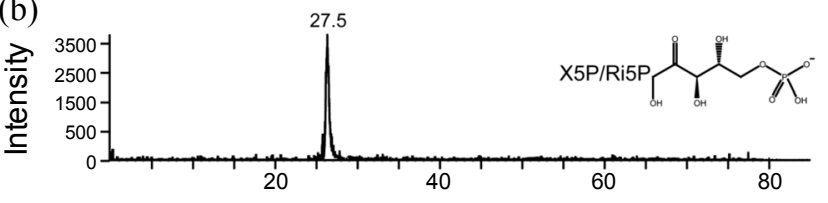

(c)

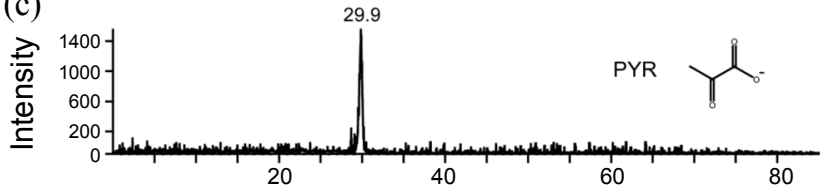

(d)

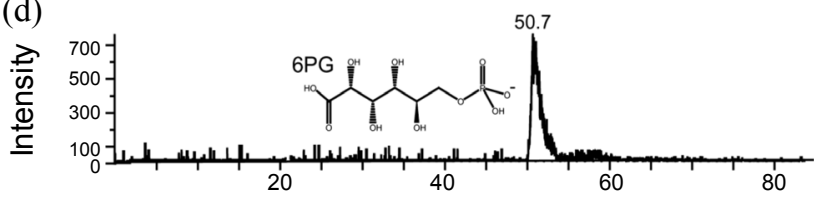

(e)

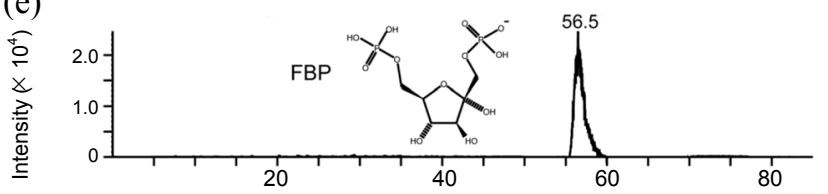

(f)

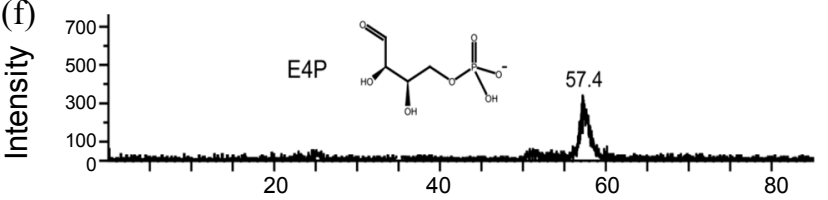

(g)

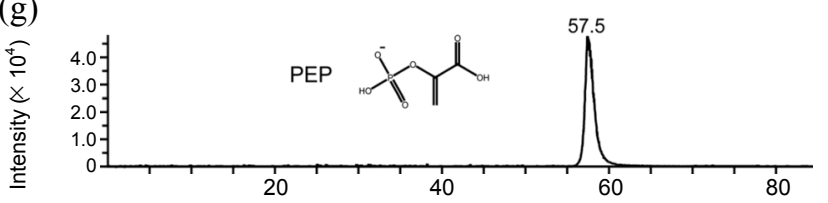

(h)

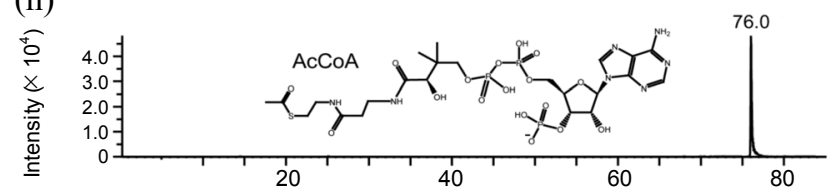

Figure 4. Single reaction monitoring (SRM) chromatogram for selective ion chromatogram: (a) G1P; (b) X5P/Ri5P; (c) PYR; (d) 6PG; (e) FBP; (f) E4P; (g) PEP; and (h) AcCoA. The concentrations of standard metabolites were as follow: G1P, $0.11 \mathrm{mg} / \mathrm{mL} ; \mathrm{X} 5 \mathrm{P} / \mathrm{Ri} 5 \mathrm{P}, 0.001$ $\mathrm{mg} / \mathrm{mL}$; PYR, $0.061 \mathrm{mg} / \mathrm{mL}$; 6 PG, $0.11 \mathrm{mg} / \mathrm{mL}$; FBP, $3.77 \mathrm{mg} / \mathrm{mL}$; E4P, $0.22 \mathrm{mg} / \mathrm{mL}$; PEP, $1.67 \mathrm{mg} / \mathrm{mL}$; AcCoA, $0.89 \mathrm{mg} / \mathrm{mL}$.

ples of the batch culture taken at $6,8,10,12,14,16$, and 18 hours are denoted by upward arrows in Figure 2. The concentrations for the nine intracellular metabolites determined by the weighted regression analysis method for the given sample volume were as follow: G1P, 0 - 122.2 $\mu \mathrm{M}$; FBP, 13.3 - $29.0 \mu \mathrm{M}$; PEP, 1.18 - $11.6 \mu \mathrm{M}$; PYR, 0 - $29.6 \mu \mathrm{M}$; AcCoA, 1.87 - $29.6 \mu \mathrm{M}$; 6PG, 0 - 69.9 $\mu \mathrm{M}$; X5P/Ri5P, 0.24 - $0.87 \mu \mathrm{M}$; E4P, 0.193 - 0.885 $\mu \mathrm{M}$. To obtain the intracellular metabolite concentrations within
Table 2. Metabolite ions monitored in the LC-MS/MS experiments: nominal $\mathrm{m} / \mathrm{z}$ values of precursor ions, [M-H] ; nominal $\mathrm{m} / \mathrm{z}$ values of main product ions; mass spectrometry experimental parameters. DP: declustering potential, CE: collision energy, CXP: collision cell exit potential

\begin{tabular}{cccccc}
\hline Metabolite & $\begin{array}{c}{[\mathrm{M}-\mathrm{H}]^{-}} \\
(\mathrm{m} / \mathrm{z})\end{array}$ & $\begin{array}{c}\text { Main product ion } \\
(\mathrm{m} / \mathrm{z})\end{array}$ & $\mathrm{DP}(\mathrm{V})$ & $\mathrm{CE}(\mathrm{V})$ & $\mathrm{CXP}(\mathrm{V})$ \\
\hline PYR & 87 & $43,-\mathrm{CO}_{2}$ & -20 & -10 & -6 \\
PEP & 167 & $79,-\mathrm{H}_{3} \mathrm{PO}_{4}$ & -50 & -50 & -6 \\
E4P & 199 & $97,\left[\mathrm{H}_{2} \mathrm{PO}_{4}\right]^{-}$ & -45 & -14 & -6 \\
Ri5P & 229 & $97,\left[\mathrm{H}_{2} \mathrm{PO}_{4}\right]^{-}$ & -20 & -16 & -6 \\
X5P & 229 & $97,\left[\mathrm{H}_{2} \mathrm{PO}_{4}\right]^{-}$ & -20 & -16 & -6 \\
G1P & 259 & $97,\left[\mathrm{H}_{2} \mathrm{PO}_{4}\right]^{-}$ & -35 & -18 & -6 \\
6PG & 275 & $79,\left[\mathrm{PO}_{3}\right]^{-}$ & -30 & -50 & -6 \\
FBP & 339 & $97,\left[\mathrm{H}_{2} \mathrm{PO}_{4}\right]^{-}$ & -27 & -30 & -6 \\
AcCoA & 808 & $79,\left[\mathrm{PO}_{3}\right]^{-}$ & -115 & -96 & -6 \\
\hline
\end{tabular}

Table 3. Limit of detection: LOD, intracellular LOD, and absolute quantities $[\mathrm{ng} / \mathrm{mL}]$ in cell extracts

\begin{tabular}{cccc}
\hline Compound & LOD $(\mu \mathrm{M})$ & Intracellular LOD $(\mu \mathrm{M})$ & LOD $(\mathrm{ng} / \mathrm{mL})$ \\
\hline G1P & $0.08 \mu \mathrm{M}$ & $2.84 \mu \mathrm{M}$ & $20 \mathrm{ng} / \mathrm{mL}$ \\
FBP & $0.17 \mu \mathrm{M}$ & $0.182 \mu \mathrm{M}$ & $0.058 \mathrm{ng} / \mathrm{mL}$ \\
PEP & $0.1 \mu \mathrm{M}$ & $5.47 \mu \mathrm{M}$ & $0.017 \mathrm{ng} / \mathrm{mL}$ \\
PYR & $2.27 \mu \mathrm{M}$ & $21.46 \mu \mathrm{M}$ & $200 \mathrm{ng} / \mathrm{mL}$ \\
AcCoA & $0.08 \mu \mathrm{M}$ & $0.311 \mu \mathrm{M}$ & $0.065 \mathrm{ng} / \mathrm{mL}$ \\
6PG & $0.24 \mu \mathrm{M}$ & $9.27 \mu \mathrm{M}$ & $67 \mathrm{ng} / \mathrm{mL}$ \\
R5P/X5P & $0.02 \mu \mathrm{M}$ & $0.022 \mu \mathrm{M}$ & $4 \mathrm{ng} / \mathrm{mL}$ \\
E4P & $0.01 \mu \mathrm{M}$ & $0.039 \mu \mathrm{M}$ & $2 \mathrm{ng} / \mathrm{mL}$ \\
\hline
\end{tabular}

the intracellular volume of $E$. coli, a correction was again made for these values to account for the varying biomass dry weights of the samples at given times (refer to Figure 2); these results are shown in Figure 5. For the correction, the value of $2.15 \mathrm{~mL} / \mathrm{g}$ dry cell mass was used for the intracellular volume of the $E$. coli cells. ${ }^{21}$ This procedure was repeated three times to obtain the average concentration of the metabolites.

Figure 5 shows the average intracellular concentration of metabolites in E. coli taken at the seven different cultivation times. Each metabolite shows quite a different concentration profile. For example, G1P, FBP, and 6PG declined fairly steadily, while PEP, PYR, AcCoA, X5P+Ri5P, and E4P showed a trend of an increase at early cultivation times, followed by a decrease, or vice versa. A distinctive range of concentrations was exhibited for each metabolite. The two extreme cases were PYR and X5P + Ri5P, which were on the order of 1 and 0.001 $\mathrm{mM}$, respectively. Specifically, G1P and 6PG had very similar concentration ranges, which spanned from 0 to $0.8 \mathrm{mM}$. For FBP, AcCoA, and PEP, the concentrations were much lower than those of G1P and 6PG. In contrast, PYR showed much higher concentrations $(0-8.65 \mathrm{mM})$. X5P/Ri5P and E4P exhibited significantly lower concentrations, which were in the range of 0 to $0.0031 \mathrm{mM}$.

To better understand the metabolic changes that occurred around $12 \mathrm{~h}$ after the initiation of batch cultivation, the concentrations of intracellular metabolites were compared to the specific growth rates of E. coli in the batch culture (Table 4). The 
(a)

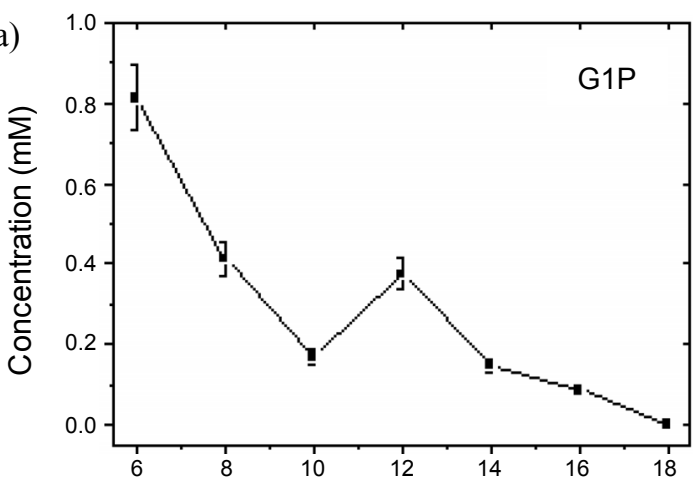

(c)

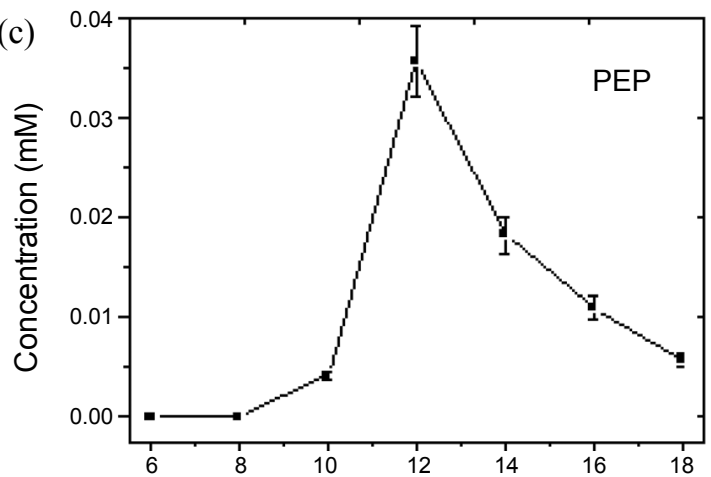

(e)

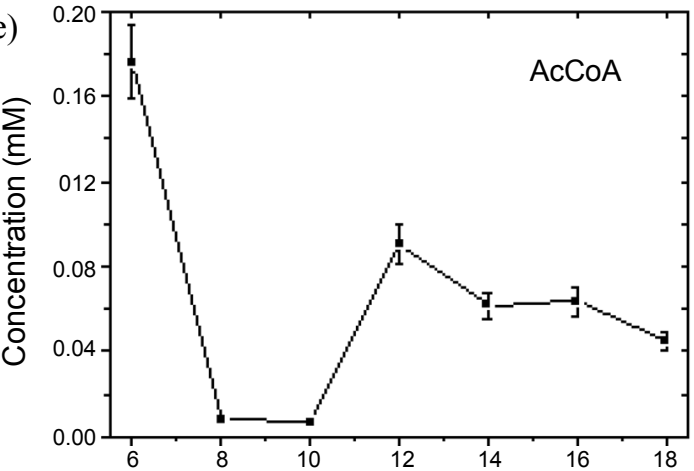

(g)

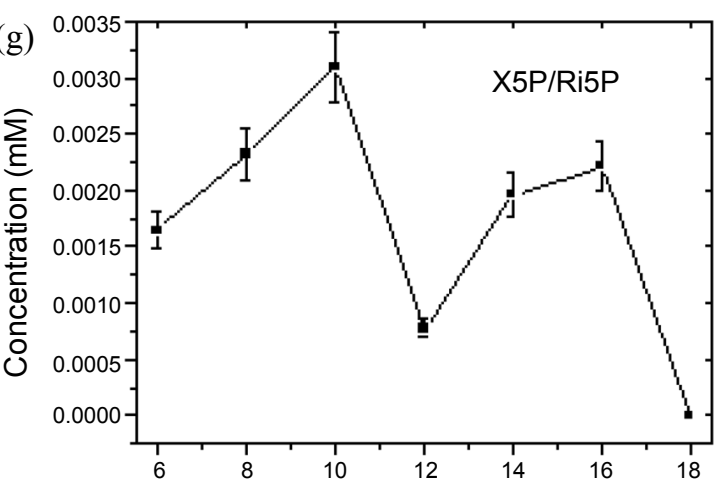

(b)

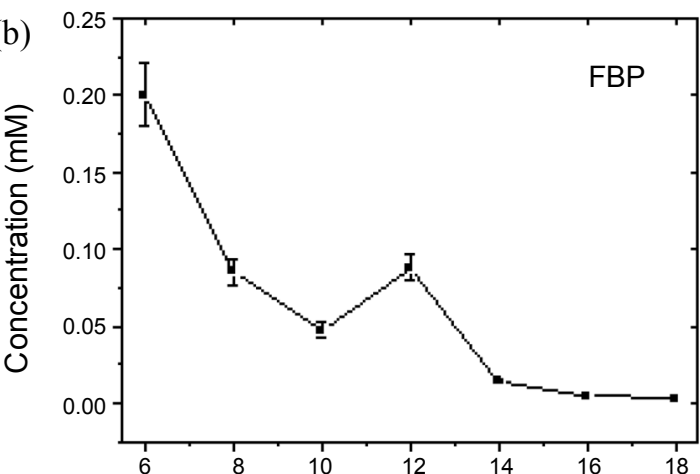

(d)

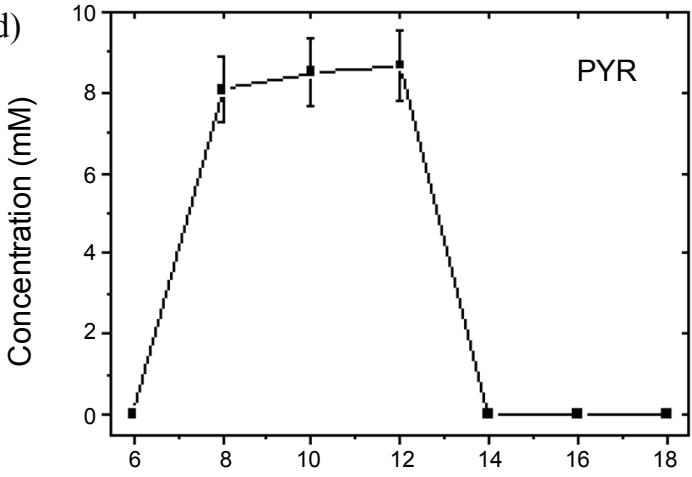

(f)

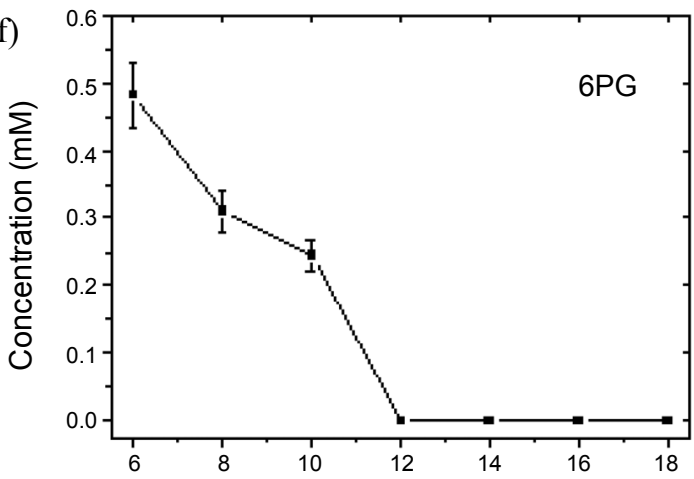

(h)

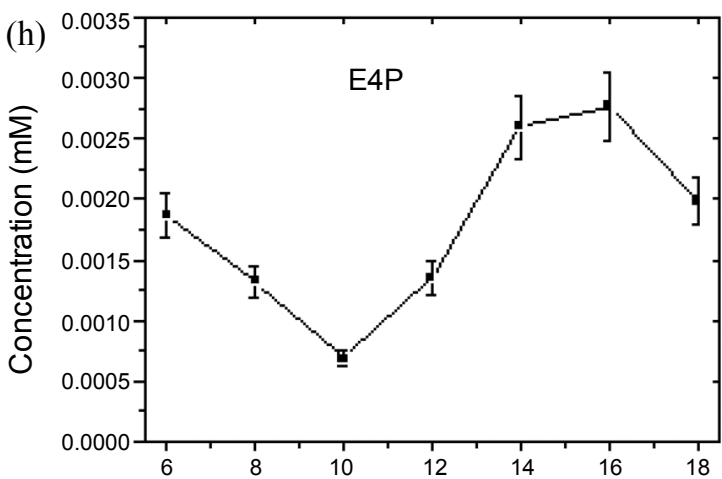

Figure 5. Cultivation time profiles of intracellular metabolite concentrations (in the intracellular volume of E. coli): (a) G1P; (b) FBP; (c) PEP; (d) PYR; (e) AcCoA; (f) 6PG; (g) X5P/Ri5P; and (h) E4P. The procedure was repeated three times to obtain the average concentration of the metabolites.

specific growth rates of the $E$. coli batch culture and the concentrations of individual intracellular metabolite were determined from Figure 2. The specific growth rate of $0.0318 \mathrm{~h}^{-1}$ occurred at $12 \mathrm{~h}$. Most intracellular metabolite concentrations decreased in the range of specific growth rates from $0.0043-0.0318 \mathrm{~h}^{-1}$, except those of E4P and X5P/Ri5P. The concentrations of E4P and $\mathrm{X} 5 \mathrm{P} / \mathrm{Ri} 5 \mathrm{P}$ increased in the range of specific growth rates from $0.0043-0.0318 \mathrm{~h}^{-1}$. The specific growth rate of $0.0043 \mathrm{~h}^{-1}$ occurred $16 \mathrm{~h}$ after the initiation of the batch culture.

Analysis of Metabolites in Two Different Growth Phases. 
Table 4. Relationship between the intracellular metabolite concentration and the specific growth rate of E. coli

\begin{tabular}{ccccccccc}
\hline Specific growth rate $\left(\mathrm{h}^{-1}\right)$ & G1P $(\mathrm{mM})$ & FBP $(\mathrm{mM})$ & PEP $(\mathrm{mM})$ & PYR $(\mathrm{mM})$ & AcCoA $(\mathrm{mM})$ & 6PG $(\mathrm{mM})$ & X5P + Ri5P $(\mathrm{mM})$ & E4P $(\mathrm{mM})$ \\
\hline-0.0704 & 0 & 0.0028 & 0.0057 & 0 & 0.0451 & 0 & 0 & 0.0019 \\
0.0043 & 0.0886 & 0.0047 & 0.0109 & 0 & 0.0634 & 0 & 0.0022 & 0.0028 \\
0.0258 & 0.1468 & 0.0143 & 0.0183 & 0 & 0.0619 & 0 & 0.0019 & 0.0026 \\
0.0318 & 0.3751 & 0.0880 & 0.0356 & 8.658 & 0.0908 & 0 & 0.0008 & 0.0014 \\
0.0603 & 0.1680 & 0.0474 & 0.0042 & 8.490 & 0.0071 & 0.2439 & 0.0031 & 0.0007 \\
0.1490 & 0.4130 & 0.0852 & 0 & 8.050 & 0.0083 & 0.3099 & 0.0023 & 0.0013 \\
0.3359 & 0.8121 & 0.2002 & 0 & 0 & 0.1760 & 0.4826 & 0.0016 & 0.0019 \\
\hline
\end{tabular}

The concentration profiles of the intracellular metabolites in this study can be related to a few metabolic pathways such as the Embden-Meyerhof pathway and the pentose phosphate pathway. The metabolites involved in the Embden-Meyerhof pathway, e.g., PEP, FBP, PYR, generally showed a remarkably different concentration profile from those involved in the pentose phosphate pathway (X5P/Ri5P, E4P). In particular, in the stationary phase, metabolites in the Embden-Meyerhof pathway exhibited a continuous decrease in concentration, while metabolites in the pentose phosphate pathway exhibited a rapid increase during the $12-14 \mathrm{~h}$ period of the stationary phase (see Figure 5).

The concentration profiles of some intracellular metabolites can be understood in relation to the metabolic pathways for the metabolites of interest. For example, PYR showed a sharp drop in concentration after $12 \mathrm{~h}$, due to the shortage of precursor metabolites, e.g., PEP, which are necessary for the formation of PYR. In the case of X5P/Ri5P and E4P, which are precursors in the amino acid synthesis pathway, the concentration profiles show an accumulation of these metabolites at $12-16 \mathrm{~h}$. This finding may be related to the previous observation that the protein synthesis pathway is inhibited at the early stage of the stationary phase of growth. ${ }^{22}$ The inhibited amino acid synthesis pathway may lead to the accumulation of precursor metabolites.

The intracellular metabolite concentration profiles shown in Figure 5 generally coincide with those of extracellular metabolites in Figure 2. For example, the intracellular PYR concentration fell sharply after $12 \mathrm{~h}$, which is consistent with the exhaustion of glucose $(\nabla)$ at $12 \mathrm{~h}$, that is, at the end of the exponential phase (see Figure 2). It was also found that AcCoA showed some residual concentrations even after $18 \mathrm{~h}$ in the stationary phase. This is consistent with the observation that the extracellular acetate concentrations showed a slow increase even in the stationary phase.

\section{Conclusion}

Metabolic regulation in the E. coli K12 strain W3110 was studied by monitoring changes in the intracellular metabolite concentrations at two different growth phases, stationary and exponential. An extraction procedure using 100\% methanol $\left(-40{ }^{\circ} \mathrm{C}\right)$ was performed to extract intracellular metabolites from E. coli. All of the quantifications of intracellular metabolites were made using the SRM mode of triple-quadrupole mass spectrometer. This method allowed for the combined separation and quantification of intracellular metabolites from the most interesting biological compounds in central carbon metabolism.
This method can be successfully applied to identify and quantify these intracellular metabolites in various cell extract samples. The intracellular metabolite concentration data can be used to help understand of microbial metabolism. This can lead to mathematical modeling based on accurate intracellular metabolite concentration measurements of the most important reactants in microbial metabolism.

Acknowledgments. This work was supported by the Korean Systems Biology Research Project (20100002164) of the Ministry of Education, Science and Technology (MEST) through the National Research Foundation of Korea.

\section{References}

1. Fiehn, O. Comparative and Functional Genomics 2001, 2, 155.

2. Fiehn, O.; Kopka, J.; Dörmann, P.; Altmann T.; Trethewey, R. N.; Willmitzer, L. Nature Biotechnol. 2000, 18, 1157.

3. Gipson, G. T.; Tatsuoka, K. S.; Sokhansanj, B. A.; Ball, R. J.; Connor, S. C. Metabolomics 2008, 4, 94

4. Hollywood, K.; Brison, D. R.; Goodacre, R. Proteomics 2006, 6, 4716.

5. Dettmer, K.; Aronov, P. A.; Hammock, B. D. Mass Spectrometry Reviews 2007, 26, 51

6. Shulaev, V. Briefings in Bioinformatics 2006, 7, 128.

7. Buchholz, A.; Hurlebaus, J.; Wandrey, C.; Takors, R. Biomolecular Engineering 2002, 19, 5.

8. Dunn, W. B.; Ellis, D. I. Trends in Analytical Chemistry 2005, 24, 285.

9. Moco, S.; Bino, R. J.; De Vos, R. C. H.; Vervoort, J. Trends in Analytical Chemistry 2007, 26, 855.

10. Luo, B.; Groenke, K.; Takors, R.; Wandrey, C.; Oldiges, M. Journal of Chromatography A 2007, 1147, 153.

11. Coulier, L.; Bas, R.; Jespersen, S.; Verheij, E.; Van der werf, M. J.; Hankemeier, T. Analytical Chemistry 2006, 78, 6573.

12. Huck, J. H. J.; Struys, E. A.; Verhoeven, N. M.; Jakobs C.; Van der knap, M. S. Clinical Chemistry 2003, 49, 1375.

13. Sekiguchi, Y.; Mitsuhashi, N.; Kokaji, T.; Miyakoda, Hi.; Mimura, T. Journal of Chromatography A 2005, 1085, 131.

14. Van dam, J. C.; Eman, M. R.; Frank, J.; Lange, H. C.; Van dedem, G. W. K.; Heijnen S. J. Anal. Chim. Acta 2002, 460, 209.

15. Chassgnolie, C.; Noisommit-Rizzi, N.; Schmid, J. W.; Mauch, K.; Reuss, M. Biotechnol. Bioeng. 2002, 79, 53.

16. Winder, C. L.; Dunn, W. B.; Schuler, S.; Broadhurst, D.; Jarvis, R.; Stephens, G. M.; Goodacre, R. Analytical Chemistry 2008, 80, 2939.

17. Maharjan, R. P.; Ferenci, T. Analytical Biochemistry 2003, 313, 145.

18. Miller, J. C.; Miller, J. N. Statistics for Analytical Chemistry; Ellis Horwood Ltd.: Chichester, UK, 1988.

19. Rao, D. G. In Introduction to Biochemical Engineering; McGrawHill: New delhi, India, 2006; p169.

20. Soga, T.; Ueno, Y.; Naraoka, H.; Ohashi, Y.; Tomita, M.; Nishioka, T. Analytical Chemistry 2002, 74, 2233.

21. Pramanik, J.; Keasling, J. D. Biotechnol Bioeng. 1997, 56, 399

22. Kolter, R.; Siegele, D. A.; Torma, A. Annual Reviews Microbiol. 1993, 47,855 . 